\title{
Interband, intraband, and excited-state direct photon absorption of silicon and germanium nanocrystals embedded in a wide band-gap lattice
}

\author{
C. Bulutay* \\ Department of Physics and Institute of Materials Science and Nanotechnology, Bilkent University, Bilkent, Ankara 06800, Turkey
}

(Received 26 May 2007; revised manuscript received 13 August 2007; published 20 November 2007)

\begin{abstract}
Embedded Si and Ge nanocrystals (NCs) in wide band-gap matrices are studied theoretically using an atomistic pseudopotential approach. Small clusters to large NCs containing the order of several thousand atoms are considered. Effective band-gap values as a function of NC diameter reproduce very well the available experimental and theoretical data. It is observed that the highest occupied molecular orbital for both $\mathrm{Si}$ and $\mathrm{Ge}$ NCs and the lowest unoccupied molecular orbital for Si NCs display oscillations with respect to size among the different irreducible representations of the $C_{3 v}$ point group to which these spherical NCs belong. Based on this electronic structure, first, the interband absorption is thoroughly studied, which shows the importance of surface polarization effects that significantly reduce the absorption when included. This reduction is found to increase with decreasing $\mathrm{NC}$ size or with increasing permittivity mismatch between the NC core and the host matrix. Reasonable agreement is observed with the experimental absorption spectra where available. The deformation of spherical NCs into prolate or oblate ellipsoids is seen to introduce no pronounced effects for the absorption spectra. Next, intraconduction and intravalence band absorption coefficients are obtained in the wavelength range from far-infrared to visible region. These results can be valuable for the infrared photodetection prospects of these NC arrays. Finally, excited-state absorption at three different optical pump wavelengths, 532, 355, and $266 \mathrm{~nm}$ are studied for 3 and $4 \mathrm{~nm}$ diameter NCs. This reveals strong absorption windows in the case of holes and a broad spectrum in the case of electrons, which can especially be relevant for the discussions on achieving gain in these structures.
\end{abstract}

DOI: $10.1103 /$ PhysRevB.76.205321

PACS number(s): 73.22.-f, 78.67.Bf, 78.40.-q

\section{INTRODUCTION}

The field of silicon and germanium nanocrystals (NCs) is very active due to important technological achievements and prospects particularly in connection with optics such as light emitting diodes and lasers. ${ }^{1-4}$ Two fundamental processes describing the interaction of light with matter are the photon absorption and emission. In the context of NCs, it has been shown both experimentally ${ }^{5}$ and theoretically ${ }^{6,7}$ that the interface properties have dramatic effects on the emission properties. On the other hand, absorption measurements are less sensitive to surface quality and allow for a more direct characterization of the intrinsic structure of NCs. ${ }^{8}$ Therefore, the study of the direct photon absorption in NCs can provide a clear physical understanding. Moreover, with the evergrowing importance of renewable energy resources, the research on the new-generation photovoltaics has gained momentum and hence the subject of direct photon absorption in nanocrystalline silicon (nc-Si). ${ }^{9}$ However, both experimentally ${ }^{10-15}$ and theoretically, ${ }^{16-23}$ researchers until now have predominantly focused on the interband absorption process. This is the only optical absorption possibility for an intrinsic semiconductor NC under equilibrium. By relaxing these two constraints, we can introduce other absorption channels, mainly through carrier injection or optical pumping. The associated absorption in either case is sometimes referred to as the "free" carrier absorption despite the carrier confinement in NCs. In our work, we discriminate between the two. The electrical injection or doping gives rise to intraband absorption, also termed as intersubband absorption, ${ }^{24}$ which has practical importance for mid- and near-infrared photodetectors. $^{25}$ The optical pumping which is usually well above the effective band gap leads to excited-state absorption (also termed as photoinduced absorption), which is an undesired effect that can inhibit the development of optical gain. ${ }^{26}$ Recent experiments on excited-state absorption concluded that more attention should be devoted to the role of the excitation conditions in the quest for the silicon laser. ${ }^{27-29}$ Therefore, the aim of this work is to provide a comprehensive theoretical account of all of these direct photon absorption mechanisms in Si and Ge NCs under various size, shape, and excitation conditions. This provides a complementary track to the existing experimental efforts where the size and shape control are currently major obstacles.

The absorption coefficient of the semiconductor NCs depends on the product of the optical transition oscillator strength and their joint density of states as well as to their volume filling factor within the matrix. Therefore, the essential decision on a theoretical study is the sophistication level of the electronic structure. The usual trade off between the computational cost and accuracy is operational. The constraints on the former are quite stringent as a $\mathrm{NC}$ including the active region of the matrix surrounding itself can contain on the order of 10000 atoms. As for the latter, not only the accuracy but also the validity of a chosen approach can become questionable. Computationally low-cost approaches such as the envelope function in conjunction with eight-band $\mathbf{k} \cdot \mathbf{p}$ are not as accurate for this task and furthermore, they miss some critical symmetries of the underlying lattice. ${ }^{30}$ On the other extreme, there lies the density-functional-theorybased $a b$ initio $\operatorname{codes}^{31}$ which have been applied to smaller NCs containing less than 1000 atoms, which still require very demanding computational resources. ${ }^{7,17-22,32}$ The ab initio analysis of larger NCs of sizes between 3 and $10 \mathrm{~nm}$ is 


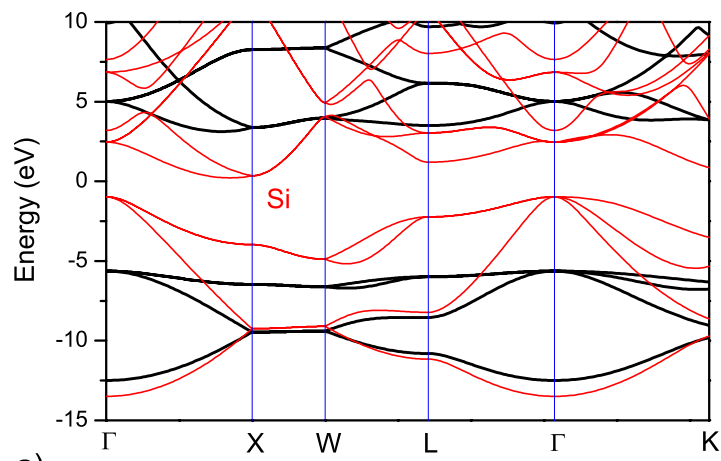

a)

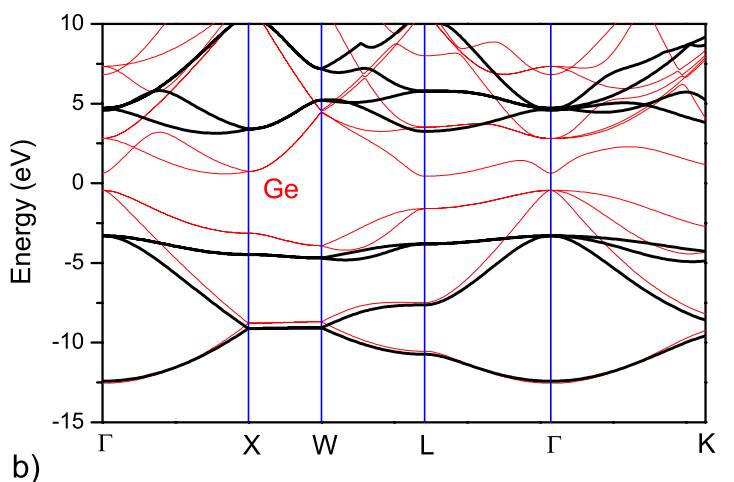

FIG. 1. (Color online) EPM band structures for bulk (a) Si and (b) Ge together with their wide band-gap matrices (thick lines), which for the former reproduces the band lineup of the $\mathrm{Si} / \mathrm{SiO}_{2}$ interface.

practically not possible with the current computer power. While this technological hurdle will be gradually overcome in the years to come, there exist other atomistic approaches that can be employed for NC research, which can be run on modest platforms and are much simpler to develop, such as the tight binding technique, which has been successfully employed by several groups. ${ }^{23,33-35}$ On the pseudopotentialbased approaches, two new recipes were proposed by Wang and Zunger over the last decade. ${ }^{36-38}$ The folded spectrum method $^{36}$ relies on the standard plane wave basis and direct diagonalization; its speed is granted from being focused on relatively few targeted states. For the study of excitons, this approach becomes very suitable, whereas for the optical absorption spectra where a large number of states contribute, it loses its advantage. The other recipe is the so-called linear combination of bulk bands (LCBBs). ${ }^{36-38}$ As a matter of fact, the idea of using bulk Bloch states in confined systems goes back to earlier times, one of its first implementations being the studies of Ninno et al. ${ }^{39,40} \mathrm{Up}$ to now, it has been used for self-assembled quantum dots, ${ }^{37,38}$ superlattices, ${ }^{41,42}$ highelectron mobility transistors, ${ }^{43}$ and very recently on the nc-Si aggregation stages. ${ }^{44}$ In this work, we apply LCBB to the electronic structure and absorption spectra of Si and Ge NCs. An important feature of this work, in contrast to commonly studied hydrogen-passivated NCs, is that we consider NCs embedded in a wide band-gap matrix, which is usually silica. ${ }^{45}$ In principle, other matrices such as alumina or silicon nitride can be investigated along the same lines.
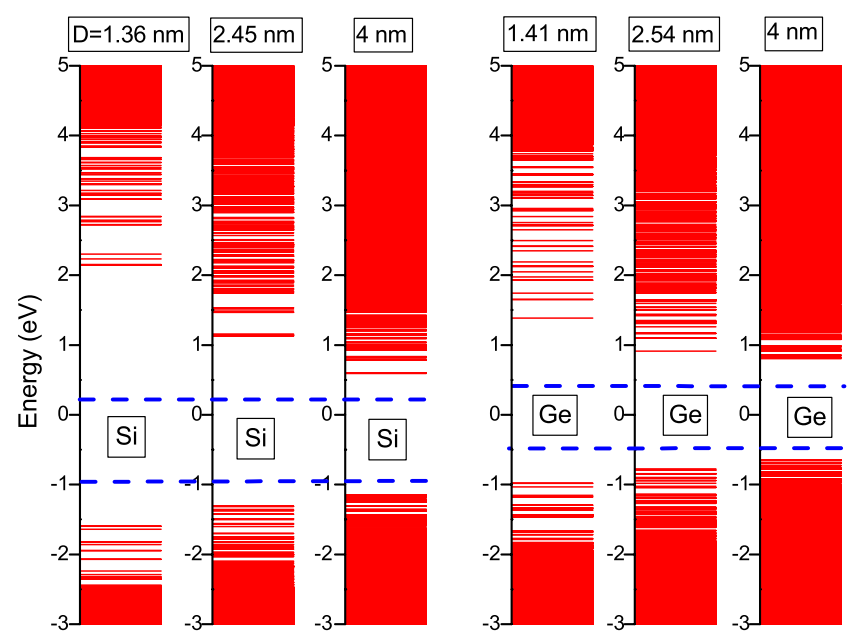

FIG. 2. (Color online) The variation of NC states with respect to diameter for Si and Ge NCs. The bulk band edges are marked with a dashed line for comparison.

The organization of the paper is as follows. In Sec. II, we describe the theoretical framework which includes some brief information on the LCBB technique and the absorption expressions. A self-critique of the theoretical model is done in Sec. III. Section IV presents the results and discussions on the band edge electronic structure, interband, intraband, and excited-state absorptions followed by our conclusions in Sec. V. Appendix section contains technical details on the employed pseudopotential form factors and our LCBB implementation.

\section{THEORY}

For the electronic structure of large-scale atomistic systems, Wang and Zunger have developed the LCBB method which is particularly convenient for embedded NCs contain-

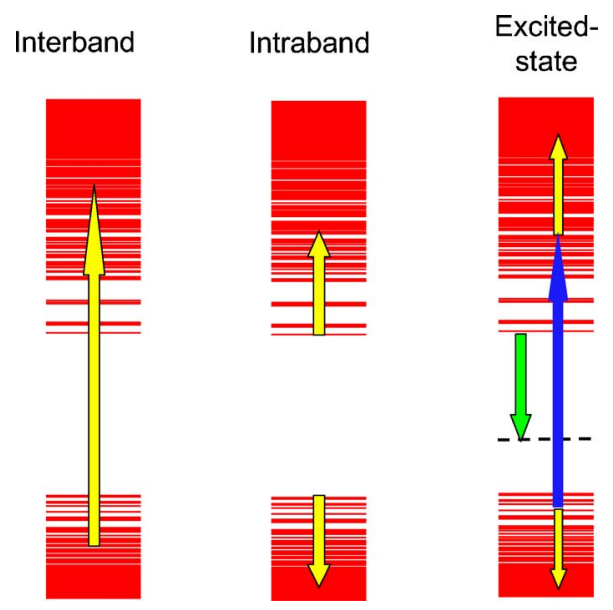

FIG. 3. (Color online) Illustration for the three different absorption processes in NCs considered in this work: interband, intraband, and excited-state absorption. The yellow (light colored) arrows indicate the direct photon absorption transitions, the blue (dark colored) arrow represents optical pumping, and the downward green arrow corresponds to luminescence, which can be to an interface state (dashed line). 

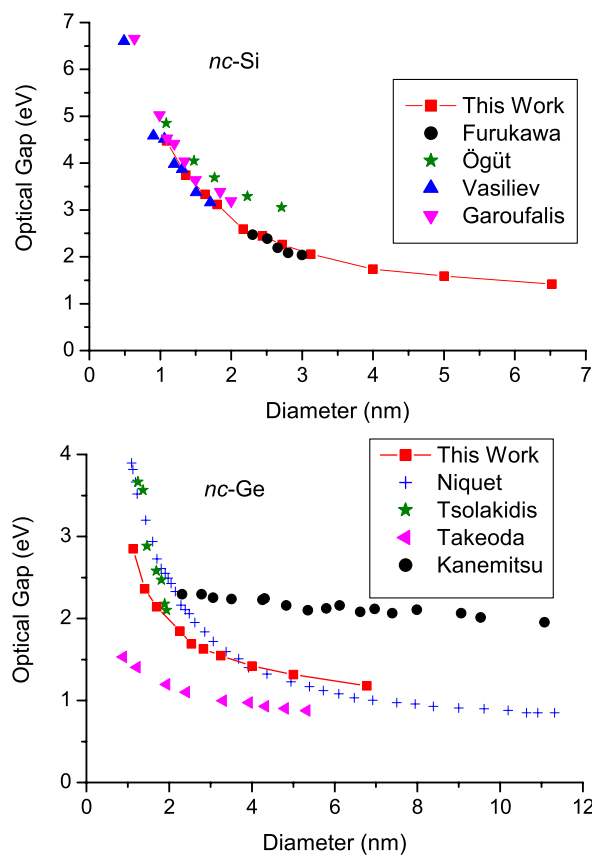

FIG. 4. (Color online) Comparison of optical gap as a function of NC diameter of this work with previous experimental and theoretical data (Refs. 10-12, 16, 22, 32, 35, and 59).

ing several thousand atoms. ${ }^{37,38}$ The fact that it is a pseudopotential-based method makes it more preferable over the empirical tight binding technique for the study of optical properties, as aimed in this work. In this technique, the NC wave function with a state label $j$ is expanded in terms of the bulk Bloch bands of the constituent core and/or embedding medium (matrix) materials,

$$
\psi_{j}(\vec{r})=\frac{1}{\sqrt{N}} \sum_{n, \vec{k}, \sigma} C_{n, \vec{k}, j}^{\sigma} e^{i \vec{k} \cdot \vec{r}} u_{n, \vec{k}}^{\sigma}(\vec{r}),
$$

where $N$ is the number of primitive cells within the computational supercell, $C_{n, \vec{k}, j}^{\sigma}$ is the expansion coefficient set to be determined, and $\sigma$ is the constituent bulk material label pointing to the NC core or embedding medium. $u_{n, \vec{k}}^{\sigma}(\vec{r})$ is the cell-periodic part of the Bloch states, which can be expanded in terms of the reciprocal lattice vectors $\{\vec{G}\}$ as

$$
u_{n, \vec{k}}^{\sigma}(\vec{r})=\frac{1}{\Omega_{0}} \sum_{\vec{G}} B_{n \vec{k}}^{\sigma}(\vec{G}) e^{i \vec{G} \cdot \vec{r}}
$$

where $\Omega_{0}$ is the volume of the primitive cell. The atomistic Hamiltonian for the system is given by

$$
\hat{H}=-\frac{\hbar^{2} \nabla^{2}}{2 m}+\sum_{\sigma, \vec{R}_{j}, \alpha} W_{\alpha}^{\sigma}\left(\vec{R}_{j}\right) v_{\alpha}^{\sigma}\left(\vec{r}-\vec{R}_{j}-\vec{d}_{\alpha}^{\sigma}\right),
$$

where $W_{\alpha}^{\sigma}\left(\vec{R}_{j}\right)$ is the weight function that takes values 0 or 1 depending on the type of atom at the position $\vec{R}_{j}-\vec{d}_{\alpha}^{\sigma}, 46$ and $v_{\alpha}^{\sigma}$ is the screened spherical pseudopotential of atom $\alpha$ of the material $\sigma$. We use semiempirical pseudopotentials for $\mathrm{Si}$ and $\mathrm{Ge}$ developed particularly for strained $\mathrm{Si} / \mathrm{Ge}$ superlat-
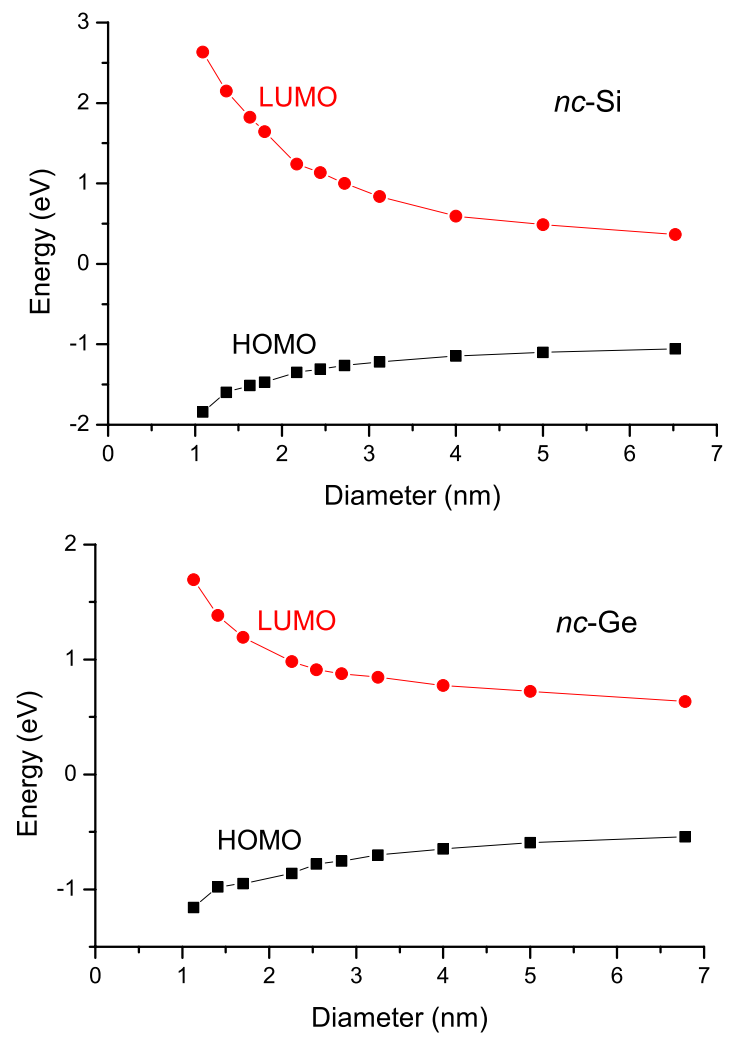

FIG. 5. (Color online) The variation of HOMO and LUMO energies with respect to $\mathrm{NC}$ diameter for $\mathrm{Si}$ and $\mathrm{Ge} \mathrm{NCs}$ that belong to the $C_{3 v}$ point group.

tices, which reproduces a large variety of measured physical data such as bulk band structures, deformation potentials, electron-phonon matrix elements, and heterostructure valence band offsets. ${ }^{47}$ With such a choice, this approach benefits from the empirical pseudopotential method (EPM), which in addition to its simplicity has another advantage over the more accurate density functional $a b$ initio techniques that run into the well-known band-gap problem, ${ }^{31}$ which is a disadvantage for the correct prediction of the excitation energies.

The formulation can be cast into the following generalized eigenvalue equation: ${ }^{38,43}$

$$
\sum_{n, \vec{k}, \sigma} H_{n^{\prime} \vec{k}^{\prime} \sigma^{\prime}, n \vec{k} \sigma} C_{n, \vec{k}}^{\sigma}=E \sum_{n, \vec{k}, \sigma} S_{n^{\prime} \vec{k}^{\prime} \sigma^{\prime}, n \vec{k} \sigma} C_{n, \vec{k}}^{\sigma}
$$

where

$$
H_{n^{\prime} \vec{k}^{\prime} \sigma^{\prime}, n \vec{k} \sigma} \equiv\left\langle n^{\prime} \vec{k} \sigma^{\prime}\left|\hat{T}+\hat{V}_{\mathrm{xtal}}\right| n \vec{k} \sigma\right\rangle
$$

$$
\left\langle n^{\prime} \vec{k} \sigma^{\prime}|\hat{T}| n \vec{k} \sigma\right\rangle=\delta_{\vec{k}^{\prime}, \vec{k}} \sum_{\vec{G}} \frac{\hbar^{2}}{2 m}|\vec{G}+\vec{k}|^{2} B_{n^{\prime} \vec{k}^{\prime}}^{\sigma^{\prime}}(\vec{G})^{*} B_{n \vec{k}}^{\sigma}(\vec{G}),
$$




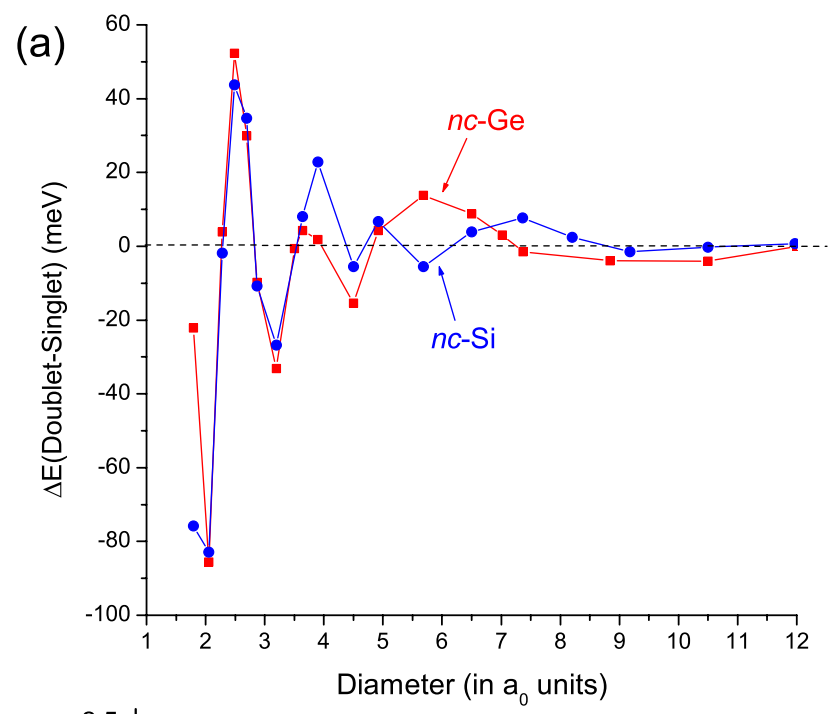

(b)

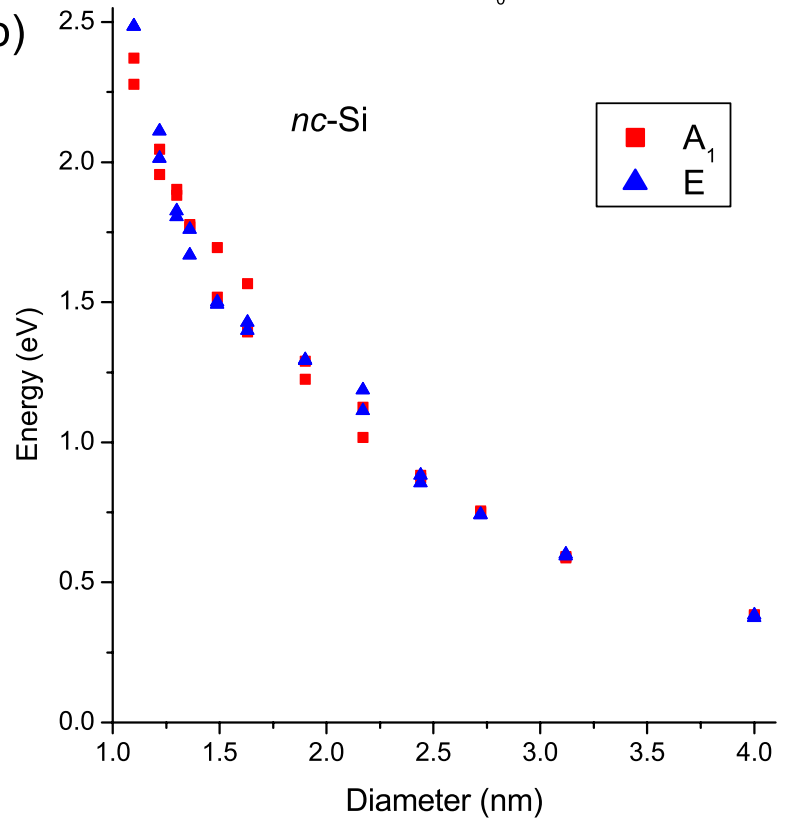

FIG. 6. (Color online) (a) The energy difference between doubly degenerate and nondegenerate states, one of which becomes the $\mathrm{HOMO}$ with respect to diameter in $\mathrm{Si}$ and Ge NCs that belong to the $C_{3 v}$ point group; solid lines are for guiding the eyes; $a_{0}$ is the lattice constant for $\mathrm{Si}$ or Ge NC. (b) The lowest three conduction states, one of which becomes the LUMO with respect to diameter in Si NCs.

$$
\begin{aligned}
&\left\langle n^{\prime} \vec{k} \sigma^{\prime}\left|\hat{V}_{\mathrm{xtal}}\right| n \vec{k} \sigma\right\rangle= \sum_{\vec{G}, \vec{G}} B_{n^{\prime} \vec{k}^{\prime}}^{\sigma^{\prime}}(\vec{G})^{*} B_{n \vec{k}}^{\sigma}(\vec{G}) \\
& \times \sum_{\sigma^{\prime \prime}, \alpha} V_{\alpha}^{\sigma^{\prime \prime}}\left(|\vec{G}+\vec{k}-\vec{G}-\vec{k}|^{2}\right) \\
& \times W_{\alpha}^{\sigma^{\prime \prime}}(\vec{k}-\vec{k}) e^{i(\vec{G}+\vec{k}-\vec{G}-\vec{k}) \cdot \vec{d}_{\alpha}^{\sigma^{\prime \prime}}} \\
& S_{n^{\prime} \vec{k}^{\prime} \sigma^{\prime}, n \vec{k} \sigma} \equiv\left\langle n^{\prime} \vec{k} \sigma^{\prime} \mid n \vec{k} \sigma\right\rangle .
\end{aligned}
$$

Here, the atoms are on regular sites of the underlying Bravais lattice: $\vec{R}_{n_{1}, n_{2}, n_{3}}=n_{1} \vec{a}_{1}+n_{2} \vec{a}_{2}+n_{3} \vec{a}_{3}$, where $\left\{\vec{a}_{i}\right\}$ are its direct
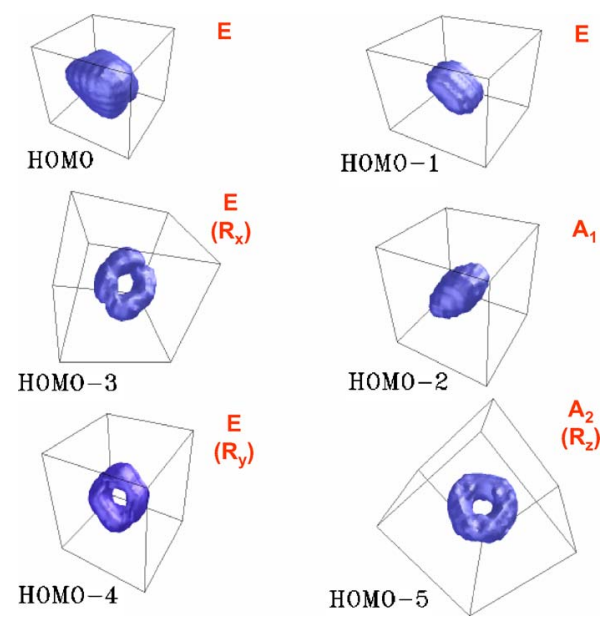

FIG. 7. (Color online) The isosurfaces of the envelopes of the wave functions of the highest-lying six valence states up to HOMO for a Si NC of diameter of $2.16 \mathrm{~nm}$. The isosurfaces are drawn for $95 \%$ of the peak value of the envelope wave functions. The $C_{3 v}$ point group representations are indicated for each wave function. Note that some of the plots are rotated with respect to others for best viewing angle.

lattice vectors of the Bravais lattice. Both the $\mathrm{NC}$ and the host matrix are assumed to possess the same lattice constant and the whole structure is within a supercell, which imposes that the periodicity condition $W\left(\vec{R}_{n_{1}, n_{2}, n_{3}}+N_{i} \vec{a}_{i}\right)=W\left(\vec{R}_{n_{1}, n_{2}, n_{3}}\right)$, recalling its Fourier representation $W\left(\vec{R}_{n_{1}, n_{2}, n_{3}}\right)$

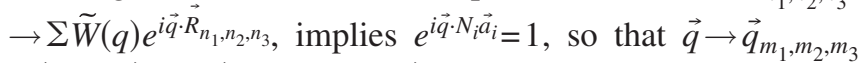
$=\vec{b}_{1} \frac{m_{1}}{N_{1}}+\vec{b}_{2} \frac{m_{2}}{N_{2}}+\vec{b}_{3} \frac{m_{3}}{N_{3}}$, where $\left\{\vec{b}_{i}\right\}$ are the reciprocal lattice vectors of the bulk material. Thus, the reciprocal space of the supercell arrangement is not a continuum but is of the grid form composed of points $\left\{\vec{q}_{m_{1}, m_{2}, m_{3}}\right\}$, where $m_{i}=0,1, \ldots, N_{i}$ -1 .

An important issue is the choice of the host matrix material. If the $\mathrm{NC}$ is surrounded by vacuum, this corresponds to the freestanding case. However, the dangling bonds of the surface NC atoms lead to quite a large number of interface states, which adversely contaminate especially the effective band-gap region of the NC. In practice, NCs are embedded into a wide band-gap host matrix, which is usually silica. ${ }^{45}$ However, the pseudopotential for oxygen is nontrivial in the case of $\mathrm{EPM}^{48}$ and, furthermore, the lattice constant of $\mathrm{SiO}_{2}$ is not matched to either of the core materials introducing strain effects. Therefore, we embed the Si and Ge NCs into an artificial wide band-gap medium, which for the former reproduces the proper band alignment of the $\mathrm{Si} / \mathrm{SiO}_{2}$ system. To circumvent the strain effects which are indeed present in the actual samples, we set the lattice constant and crystal structure of the matrix equal to that of the core material. The pseudopotential form factors of the wide band-gap matrices for $\mathrm{Si}$ and $\mathrm{Ge}$ can easily be produced starting from those of the core materials. More details are provided in the Appendix. The resultant bulk band structures for $\mathrm{Si}$ and $\mathrm{Ge}$ and their host wide band-gap matrices are shown in Fig. 1. With the use of such a lattice-matched matrix providing the perfect termination of the surface bonds of the NC core atoms leads 
TABLE I. $C_{3 v}$ irreducible representations of the HOMO and LUMO in $\mathrm{Si}$ and $\mathrm{Ge} \mathrm{NCs}$ of various diameters $(D)$.

\begin{tabular}{|c|c|c|c|c|c|c|}
\hline \multirow[b]{2}{*}{$N_{\text {core }}$} & \multicolumn{3}{|c|}{ nc-Si } & \multicolumn{3}{|c|}{ nc-Ge } \\
\hline & $\begin{array}{c}D \\
(\mathrm{~nm})\end{array}$ & HOMO & LUMO & $\begin{array}{c}D \\
(\mathrm{~nm})\end{array}$ & HOMO & LUMO \\
\hline 32 & 1.06 & $A_{1}$ & $A_{1}$ & 1.11 & $A_{1}$ & $A_{1}$ \\
\hline 38 & 1.13 & $A_{1}$ & $A_{1}$ & 1.18 & $A_{1}$ & $A_{1}$ \\
\hline 56 & 1.29 & $A_{1}$ & $A_{1}$ & 1.34 & $A_{1}$ & $A_{1}$ \\
\hline 74 & 1.41 & $E$ & $E$ & 1.47 & $E$ & $A_{1}$ \\
\hline 86 & 1.49 & $E$ & $E$ & 1.55 & $E$ & $A_{1}$ \\
\hline 116 & 1.64 & $A_{1}$ & $E$ & 1.71 & $A_{1}$ & $A_{1}$ \\
\hline 130 & 1.71 & $E$ & $A_{1}$ & 1.78 & $A_{2}$ & $A_{1}$ \\
\hline 136 & 1.73 & $A_{1}$ & $E$ & 1.80 & $A_{1}$ & $A_{1}$ \\
\hline 190 & 1.94 & $A_{1}$ & $A_{1}$ & 2.01 & $A_{1}$ & $A_{1}$ \\
\hline 264 & 2.16 & $E$ & $A_{1}$ & 2.25 & $E$ & $A_{1}$ \\
\hline 384 & 2.45 & $A_{1}$ & $E$ & 2.55 & $A_{2}$ & $A_{1}$ \\
\hline 522 & 2.71 & $E$ & $E$ & 2.82 & $E$ & $A_{1}$ \\
\hline 690 & 2.98 & $A_{1}$ & $A_{1}$ & 3.10 & $A_{2}$ & $A_{1}$ \\
\hline 768 & 3.08 & $A_{1}$ & $A_{1}$ & 3.21 & $E$ & $A_{1}$ \\
\hline 1702 & 4.02 & $E$ & $E$ & 4.18 & $A_{2}$ & $A_{1}$ \\
\hline
\end{tabular}

to the removal of all gap states, as can be observed in Fig. 2. In these plots, the evolution of the effective band gaps toward their bulk values (marked by dashed lines) is clearly seen as the diameter increases.

Once the electronic wave functions of the NCs are available, their linear optical properties can be readily computed. The three different types of direct (zero phonon) photon absorption processes considered in this work are illustrated in Fig. 3. These are interband, intraband, and excited-state absorptions. In the latter, the blue (dark colored) arrow represents optical pumping and following carrier relaxation, the downward green arrow corresponds to luminescence, which can be to a final interface state (dashed line). ${ }^{2}$ For all these processes, the relevant quantity is the imaginary part of the dielectric function. Within the independent-particle approximation and the artificial supercell framework, ${ }^{17}$ it becomes

$$
\operatorname{Im}\left\{\boldsymbol{\epsilon}_{a a}(\omega)\right\}=\frac{(2 \pi e \hbar)^{2}}{m_{0} V_{\mathrm{SC}}} \sum_{c, v} \frac{f_{c v}^{a a}}{E_{c}-E_{v}} \frac{\Gamma /(2 \pi)}{\left[E_{c}-E_{v}-\hbar \omega\right]^{2}+(\Gamma / 2)^{2}},
$$

where $a=x, y, z$ denotes the Cartesian components of the dielectric tensor and

$$
f_{c v}^{a a}=\frac{2 m_{0}\left|\left\langle c\left|\frac{p_{a}}{m_{0}}\right| v\right\rangle\right|^{2}}{E_{c}-E_{v}}
$$

is the oscillator strength of the transition. In these expressions, $m_{0}$ is the free electron mass, $e$ is the magnitude of the electronic charge, and $\Gamma$ is the full width at half maximum value of the Lorentzian broadening. The label $v(c)$ correspond to occupied (empty) valence (conduction) states refer- ring only to their orbital parts in the absence of spin-orbit coupling; the spin summation term is already accounted in the prefactor of Eq. (5). Finally, $V_{\mathrm{SC}}$ is the volume of the supercell, which is a fixed value chosen conveniently large to accommodate the NCs of varying diameters; however, if one uses instead, that of the $\mathrm{NC}, V_{\mathrm{NC}}$, this corresponds calculating $\operatorname{Im}\left\{\epsilon_{a a}\right\} / f_{v}$, where $f_{v}=V_{\mathrm{NC}} / V_{\mathrm{SC}}$ is the volume filling ratio of the NC. For the sake of generality, this is the form we shall be presenting our results. The electromagnetic intensity absorption coefficient $\alpha(\omega)$ is related to the imaginary part of the dielectric function through ${ }^{49}$

$$
\operatorname{Im}\left\{\epsilon_{a a}(\omega)\right\}=\frac{n_{r} c}{\omega} \alpha_{a a}(\omega),
$$

where $n_{r}$ is the index of refraction and $c$ is the speed of light.

In the case of intraband absorption, its rate depends on the amount of excited carriers. Therefore, we consider the absorption rate for one excited electron or hole that lies at an initial state $i$ with energy $E_{i}$. As there are a number of closely spaced such states, we perform a Boltzmann averaging over these states as $e^{-\beta E_{i}} / \Sigma_{j} e^{-\beta E_{j}}$. We further assume that the final states have no occupancy restriction, which can easily be relaxed if needed. The expression for the absorption rate per an excited carrier in each $\mathrm{NC}$ becomes

$$
\begin{aligned}
\frac{\alpha_{a a}}{f_{v}}= & \frac{\pi e^{2}}{2 m_{0} c n_{r} \omega V_{\mathrm{NC}}} \sum_{i, f} \frac{e^{-\beta E_{i}}}{\sum_{j} e^{-\beta E_{j}}} f_{f i}^{a a}\left[E_{f}-E_{i}\right] \\
& \times \frac{\Gamma /(2 \pi)}{\left[E_{f}-E_{i}-\hbar \omega\right]^{2}+(\Gamma / 2)^{2}},
\end{aligned}
$$

where again $a$ is the light polarization direction. 

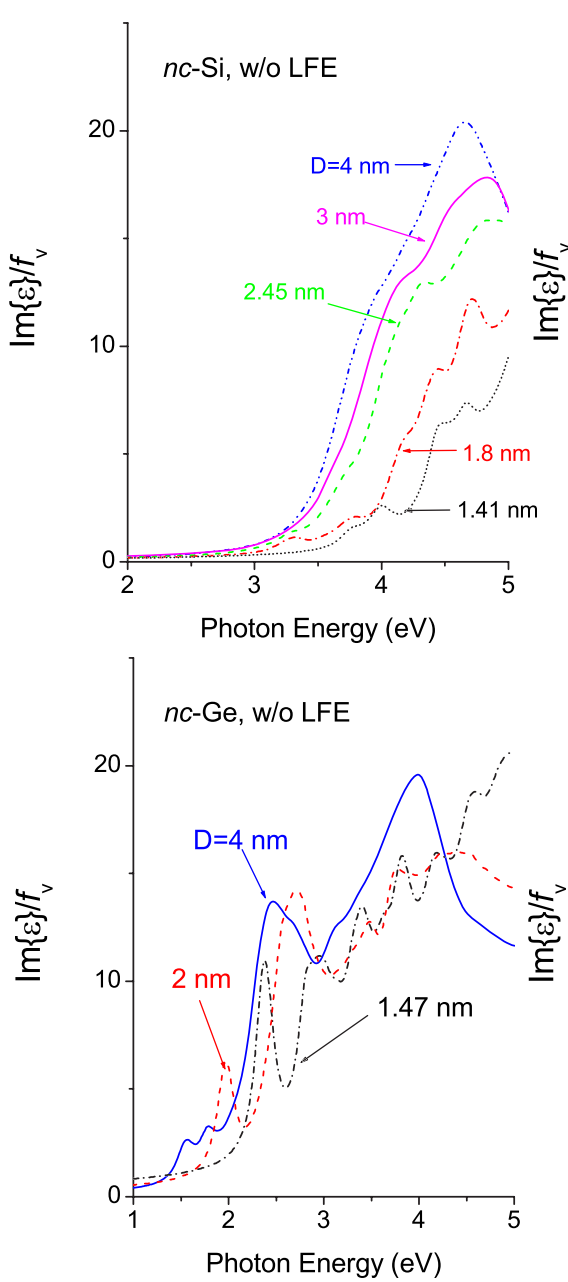
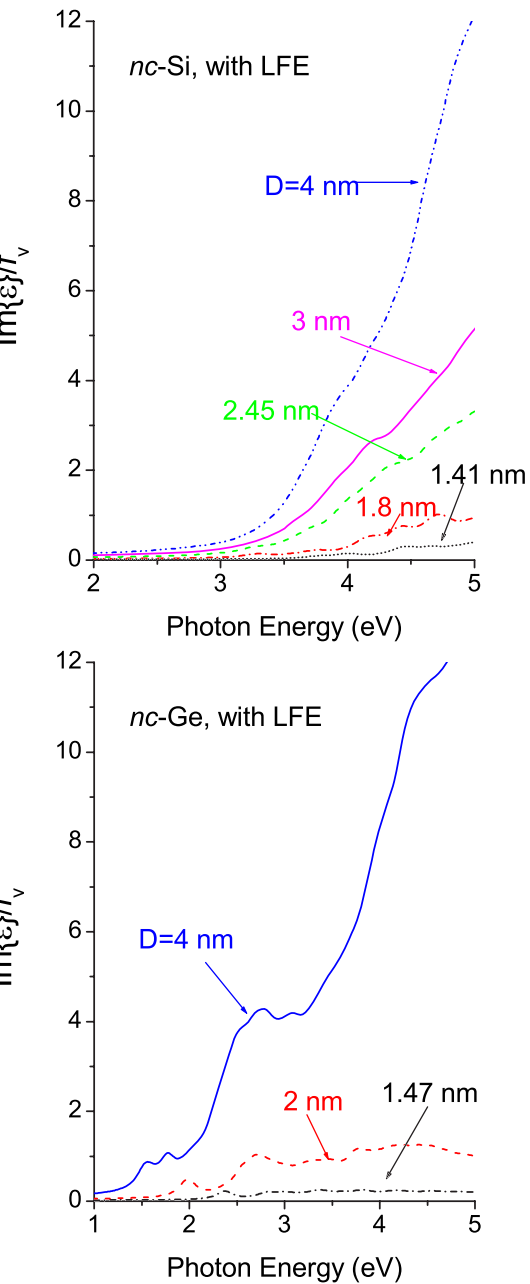

FIG. 8. (Color online) The imaginary part of dielectric function for unity volume filling factor, $f_{v}$ for $\mathrm{Si}$ and $\mathrm{Ge} \mathrm{NCs}$ at different diameters with (right panel) and without (left panel) local field effects. A Lorentzian broadening energy width of $200 \mathrm{meV}$ is used.
Finally, we include the surface polarization effects, also called local field effects (LFEs) using a simple semiclassical model, which agrees remarkably well with more rigorous treatments. ${ }^{50}$ We give a brief description of its implementation. First, using the expression

$$
\epsilon_{\mathrm{SC}}=f_{v} \epsilon_{\mathrm{NC}}+\left(1-f_{v}\right) \epsilon_{\text {matrix }},
$$

we extract (i.e., deembed) the size-dependent $\mathrm{NC}$ dielectric function $\epsilon_{\mathrm{NC}}$, where $\epsilon_{\mathrm{SC}}$ corresponds to Eq. (5), suppressing the Cartesian indices. $\epsilon_{\text {matrix }}$ is the dielectric function of the host matrix; for simplicity, we set it to the permittivity value of $\mathrm{SiO}_{2}$, i.e., $\epsilon_{\text {matrix }}=4$. Since the wide band-gap matrix introduces no absorption up to an energy of about $9 \mathrm{eV}$, we can approximate $\operatorname{Im}\left\{\epsilon_{\mathrm{NC}}\right\}=\operatorname{Im}\left\{\epsilon_{\mathrm{SC}}\right\} / f_{v}$. One can similarly obtain $\operatorname{Re}\left\{\epsilon_{\mathrm{NC}}\right\}$ within the random phase approximation, ${ }^{23}$ hence get the full complex dielectric function $\epsilon_{\mathrm{NC}}$. According to the classical Clausius-Mossotti approach, which is shown to work also for $\mathrm{NCs},{ }^{51}$ the dielectric function of the $\mathrm{NC}$ is modified as

$$
\epsilon_{\mathrm{NC}, \mathrm{LFE}}=\epsilon_{\text {matrix }}\left[\frac{4 \epsilon_{\mathrm{NC}}-\epsilon_{\text {matrix }}}{\epsilon_{\mathrm{NC}}+2 \epsilon_{\text {matrix }}}\right],
$$

to account for LFE. The corresponding supercell dielectric function $\epsilon_{\mathrm{SC} \text {,LFE }}$ follows using Eq. (9). Similarly, the intensity absorption coefficients are also modified due to surface po- larization effects, cf. Eq. (7). Its consequences will be reported in Sec. IV.

\section{SELF-CRITIQUE OF THE THEORETICAL MODEL}

The most crucial simplification of our model is the fact that strain-related effects are avoided, a route which is shared by other theoretical works. ${ }^{17,18,23,35,52}$ For large NCs, this may not be critical; however, for very small sizes, this simplification is questionable. An important support for our act is that Weissker et al. have concluded that while there is some shift and possibly a redistribution of oscillator strengths after ionic relaxation, the overall appearance of the absorption spectra does not change strongly. ${ }^{19}$ We should mention that Wang and Zunger have offered a recipe for including strain within the LCBB framework; however, this is considerably more involved. ${ }^{38}$ Another widespread simplification on $\mathrm{Si}$ and Ge NCs is the omission of the spin-orbit coupling and the nonlocal (angular momentum dependent) pseudopotential terms in the electronic structure Hamiltonian. Especially, the former is not significant for Si which is a light atom but it can have a quantitative impact on the valence states of Ge NCs; such a treatment is available in Ref. 53.

On the dielectric response, there are much more sophisticated and involved treatments, ${ }^{54}$ whereas ours is equivalent 


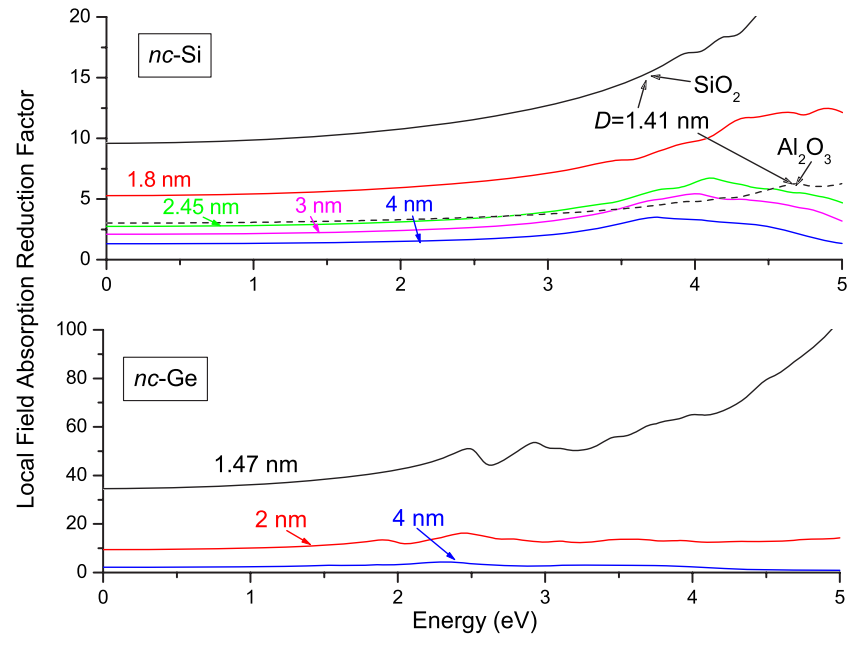

FIG. 9. (Color online) The local field absorption factor extracted from the previous figure.

to the independent-particle random phase approximation ${ }^{55}$ of the macroscopic dielectric function with the surface polarization effects included within the classical Clausius-Mossotti model. ${ }^{50}$ The contribution of the excluded excitonic and other many-body effects beyond the mean-field level can be assessed $a$ posteriori by comparing with the available experimental data. However, it is certain that the precedence should be given to classical electrostatics for properly describing the background dielectric mismatch between the core and the wide band-gap matrix. ${ }^{50}$ In our treatment, this is implemented at an atomistic level.

Another effect not accounted in this work is the role of the interface region. Our wide band-gap matrix can reproduce the proper band alignment and dielectric confinement of an $\mathrm{SiO}_{2}$ matrix; however, the interface chemistry such as silicon-oxygen double bonds ${ }^{7}$ is not represented. These were shown to be much more effective on the emission spectra. ${ }^{6,7}$ Nevertheless, our results can be taken as the benchmark for the performance of the atomistic quantum and dielectric confinement with a clean and inert interface. Finally, we do not consider the phonon-assisted ${ }^{56}$ or nonlinear absorption. The list of these major simplifications also suggests possible improvements of this work.

\section{RESULTS AND DISCUSSIONS}

In this section, we present our theoretical investigation of the linear optical properties of Si and Ge NCs. Three different direct photon absorption processes are considered, as illustrated in Fig. 3, each of which can serve for technological applications as well as to our basic understanding. However, we first begin with the dependence of the optical gap on the NC size, mainly as a check of our general framework. There exist two different atomic arrangements of a spherical NC depending on whether the center of the $\mathrm{NC}$ is an atomic position or a tetrahedral interstitial location; under no ionic relaxation, Delley and Steigmeier have treated both of these classes as having the $T_{d}$ point symmetry. ${ }^{52}$ However, the tetrahedral interstitial-centered arrangement should rather have a)

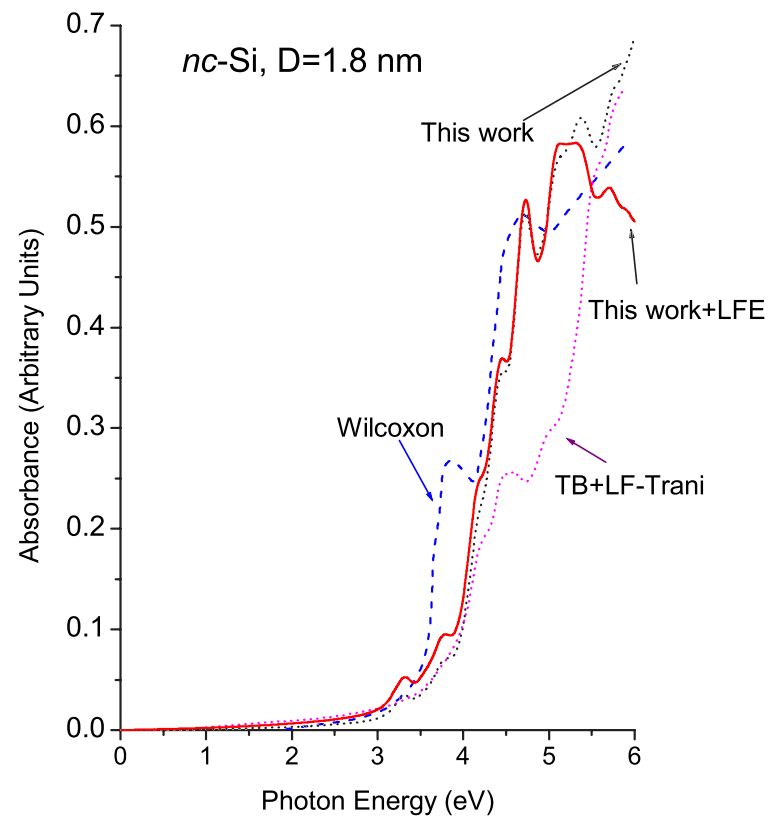

b)

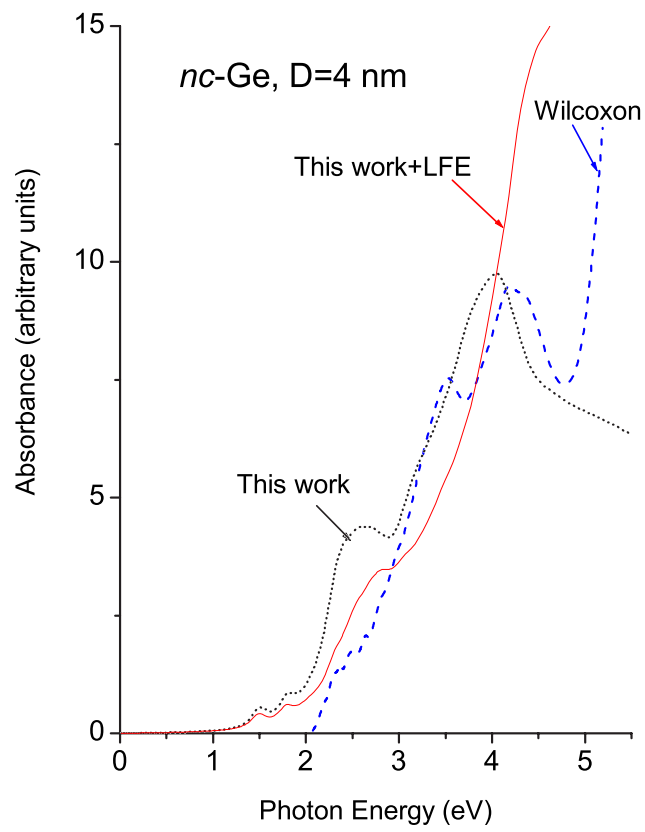

FIG. 10. (Color online) Comparison of our absorbance results with the available data: for $\mathrm{Si}$, experimental work of Wilcoxon et al. (Ref. 13) and the theoretical tight binding results of Trani et al. (Ref. 50), and for Ge, the experimental work of Wilcoxon et al. (Ref. 15). For our spectra, a Lorentzian broadening energy width of $200 \mathrm{meV}$ is used.

the lower point symmetry of $C_{3 v}$ and it is the arrangement that we construct our NCs. This leads to the even number of $\mathrm{NC}$ core atoms, whereas it becomes an odd number with the $T_{d}$ point symmetry. We identify the irreducible representation of a chosen NC state by checking its projection to the subspace of each representation. ${ }^{57}$ For the $C_{3 v}$ point group, these are denoted by $A_{1}, A_{2}$, and $E$. We utilize this group-theoretic analysis in the next subsections. 


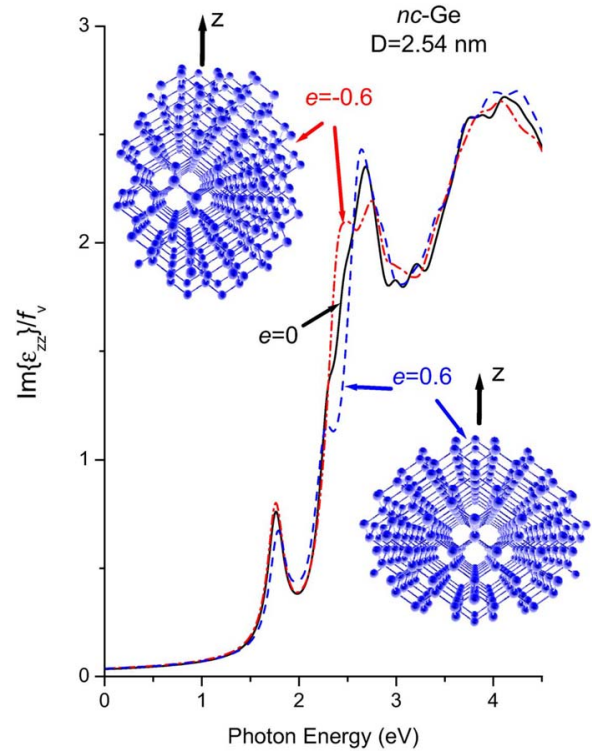

FIG. 11. (Color online) The effect of ellipticity $e$ on the $\operatorname{Im}\left\{\epsilon_{z z}\right\} / f_{v}$ for a $\mathrm{Ge} \mathrm{NC}$ with a diameter of $2.54 \mathrm{~nm}$. The insets show NC core atoms of the prolate $(e=-0.6)$ and oblate $(e=0.6)$ shapes; the $z$ direction is also indicated. A Lorentzian broadening energy width of $200 \mathrm{meV}$ is used.

\section{A. Effective optical gap}

The hallmark of quantum size effect in NCs has been the effective optical gap with quite a number of theoretical ${ }^{16,22,23,32,58,59}$ and experimental ${ }^{11-13,15}$ studies performed within the last decade. Figure 4 contains a compilation of some representative results. For $\mathrm{Si} \mathrm{NCs}$, it can be observed that there is a good agreement among the existing data, including ours. On the other hand, for the case of $\mathrm{Ge}$ $\mathrm{NCs}$, there is a large spread between the experimental data, whereas our theoretical results are in very good agreement with both $a b$ initio $^{22}$ and tight binding results. ${ }^{23}$ In our approach, the optical gap directly corresponds to the lowest unoccupied molecular orbital-highest occupied molecular orbital (LUMO-HOMO) energy difference, as calculated by the single-particle Hamiltonian in Eq. (3). This simplicity relies on the finding of Delerue et al. that the self-energy and Coulomb corrections almost exactly cancel each other for $\mathrm{Si}$ NCs larger than a diameter of $1.2 \mathrm{~nm}^{60}$

\section{B. Highest occupied molecular orbital and lowest unoccupied molecular orbital oscillations with respect to size}

When we plot the variation of individual LUMO and HOMO levels as in Fig. 5, we observe with the exception of nc-Ge LUMO curve some nonsmooth behavior that gets pronounced toward smaller sizes. The triple degeneracy in the absence of spin-orbit coupling of the valence band maximum in bulk $\mathrm{Si}$ and $\mathrm{Ge}$ is lifted into two degenerate and one nondegenerate states. The energy difference between these two sets of states is observed to display an oscillatory behavior as the NC size gets smaller, as shown in Fig. 6(a). Using the $C_{3 v}$ point group symmetry operations, we identify the doubly degenerate states to belong to $E$ representation and nonde- generate state to $A_{1}$ or $A_{2}$. Furthermore, we observe a similar oscillation in the LUMO region of Si NCs, as shown in Fig. 6(b). The low-lying conduction states of Si NCs form sixpack groups, which is inherited from the six equivalent $0.85 X$ conduction band minima of bulk Si. The confinement marginally lifts the degeneracy by sampling contributions from other parts of the Brillouin zone. This trend is observed in Fig. 6(b) as the NC size gets smaller. On the other hand, for Ge NCs, all LUMO states belong to the same $A_{1}$ representation and therefore show no oscillations (cf. Fig. 5). Ultimately, the source of these oscillations is the variation of the asphericity of the NCs of $C_{3 v}$ point symmetry with respect to size, which can energetically favor one of the closely spaced states. In the case of the LUMO state of Ge NCs, there is a substantial energy gap between LUMO and the next higher-lying state.

For further insight, we display in Fig. 7 the isosurface plots of the envelope of the six highest states up to HOMO for a Si NC of diameter $2.16 \mathrm{~nm}$. Point group representation of each state is also indicated. For this particular diameter, HOMO has $E$ representation, which is twofold degenerate. The nondegenerate $A_{1}$ state also becomes the HOMO for different diameters. This is illustrated in Table I, which shows the evolution of the HOMO and LUMO symmetries as a function of diameter for $\mathrm{Si}$ and Ge NCs. There, it can be observed that for the latter, the HOMO can also acquire the $A_{2}$ for larger diameters.

\section{Interband absorption}

The interband absorptions of Si and Ge NCs for a variety of diameters are shown in Fig. 8. For a fair comparison, all different size NCs should possess the same volume filling factor. Therefore, we display the results at unity volume filling or equivalently per $f_{v} \cdot{ }^{18}$ The left and right panels display the cases without and with surface polarization effects (or LFE), respectively. There exist remarkable differences between the two for both $\mathrm{Si}$ and Ge NCs. For instance, even though Ge NCs do not show significant size dependence without LFE, this is not the case when LFE is included. From the ratio of both panels, the so-called local field absorption reduction factor can be extracted, as shown in Fig. 9. It can be observed that its size dependence is much stronger than the energy dependence. This reduction in the absorption due to LFE can become a major concern for solar cell applications. It needs to be mentioned that this effect is highly sensitive to the permittivity mismatch between the core and matrix media. To illustrate this point, in Fig. 9, the case for $\mathrm{Al}_{2} \mathrm{O}_{3}$ matrix (having a permittivity of 9.1) is also

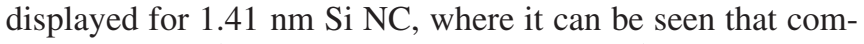
pared to $\mathrm{SiO}_{2}$ (with a permittivity of about 4), the reduction in absorption due to LFE is much less. Based on this finding, we employ these size-dependent absorption reduction factors in the results to follow including the intraband and excitedstate cases.

In Fig. 10, we compare our results with the experimental data of Wilcoxon et al. for Si NCs ${ }^{13}$ and Ge NCs. ${ }^{15}$ There is a good overall agreement in both cases especially with LFE; however, for the case of Si NCs, this is much more satisfac- 

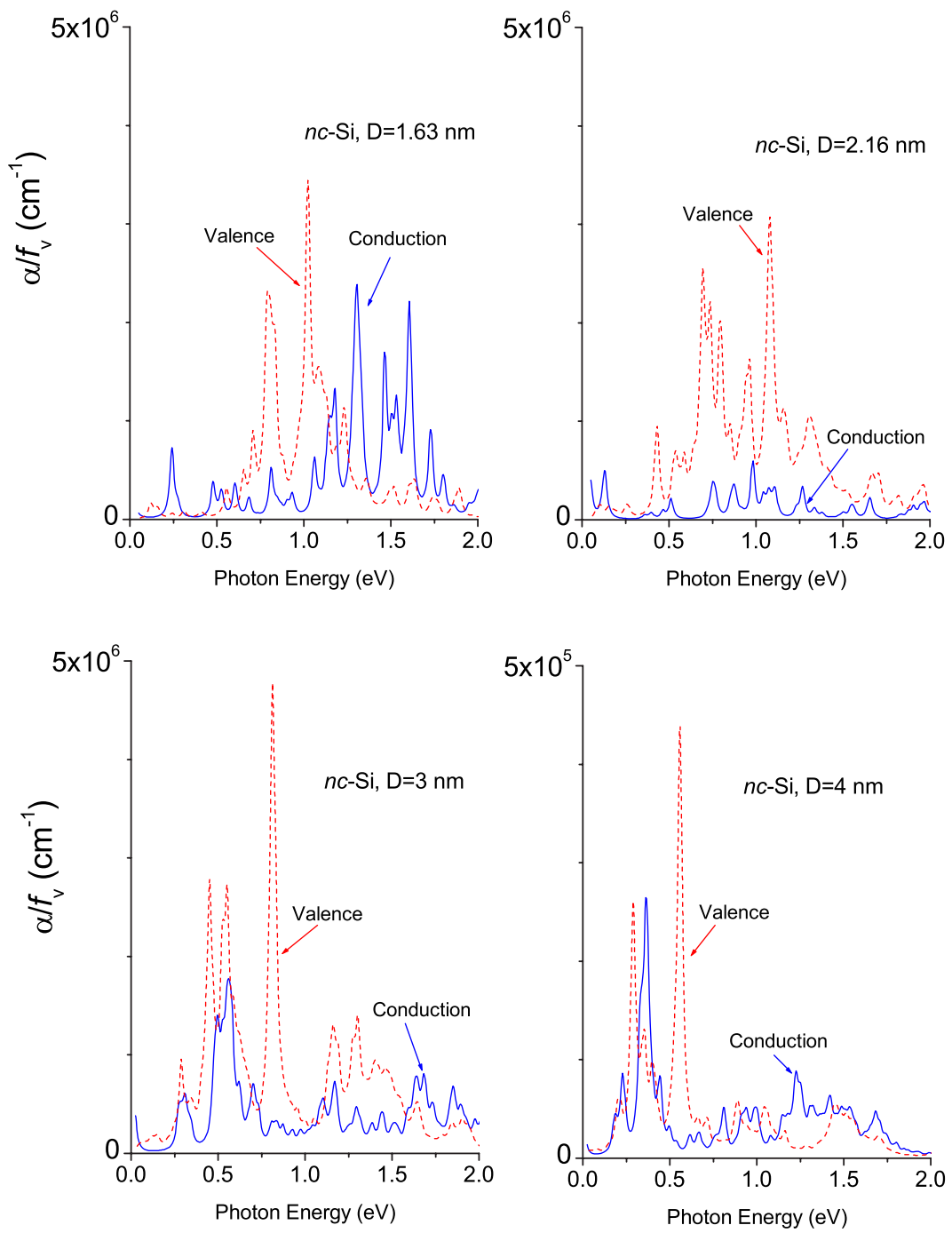

FIG. 12. (Color online) Intravalence and intraconduction state absorption coefficients in $\mathrm{Si}$ $\mathrm{NCs}$ of different diameters per excited carrier and at unity filling factor. A Lorentzian broadening energy width of $30 \mathrm{meV}$ is used. Mind the change in the vertical scale for the $4 \mathrm{~nm}$ diameter case. tory. The major discrepancies can be attributed to excitonic effects that are not included in our work. In the case of $\mathrm{Si}$ NCs [Fig. 10(a)], we also display the tight binding result of Trani et al., which also includes LFE. ${ }^{50}$

An issue of practical concern is the effect of deviation from the spherical shape of the NCs depending on the growth conditions. At this point, we would like to investigate the effect of shape anisotropy on the interband absorption. Starting from a spherical $2.54 \mathrm{~nm}$ diameter Ge NC, we form prolate and oblate ellipsoidal NCs with ellipticities $e=-0.6$ and +0.6 , respectively. All three NCs contain the same number of 384 core atoms; the atomic arrangement of the ellipsoidal NCs are shown in the inset of Fig. 11. In the same figure, we compare the $z z$ components of the imaginary part of the dielectric tensor for three different ellipticities. It is observed that the effect on the interband absorption is not significant; the difference is even less for the Si NCs (not shown).

\section{Intraband absorption}

Unlike the interband case, for the intraband absorption, we need to introduce electrons to the conduction states or holes to the valence states by an injection mechanism. We assume that after injection, these carriers relax to their respective band edges and attain a thermal distribution. Therefore, we perform a Boltzmann averaging at room temperature $(300 \mathrm{~K})$ over the initial states around LUMO (HOMO) for electrons (holes). The absorption coefficients to be presented are for unity volume filling factors and for one carrier per NC; they can easily be scaled to a different average number of injected carriers and volume filling factors. In Fig. 12, the Si NCs of different diameters are compared. The intraband absorption is observed to be enhanced as the NC size grows up to about $3 \mathrm{~nm}$ followed by a drastic fall for larger sizes. For both holes and electrons, a very large number of absorption peaks are observed from 0.5 to $2 \mathrm{eV}$. Recently, de Sousa et al. have also considered the intraband absorption in Si NCs using the effective mass approximation and taking into account the multivalley anisotropic band structure of Si. ${ }^{61}$ However, their absorption spectra lacks much of the features seen in Fig. 12. Turning to Ge NCs, shown in Fig. 13 , the intravalence band absorption profile is very similar to that of Si NCs; however, in this, case the intraconduction band absorption is much weaker.

Mimura et al. have measured the optical absorption in heavily phosphorus doped Si NCs of a diameter of $4.7 \mathrm{~nm} .^{62}$ 

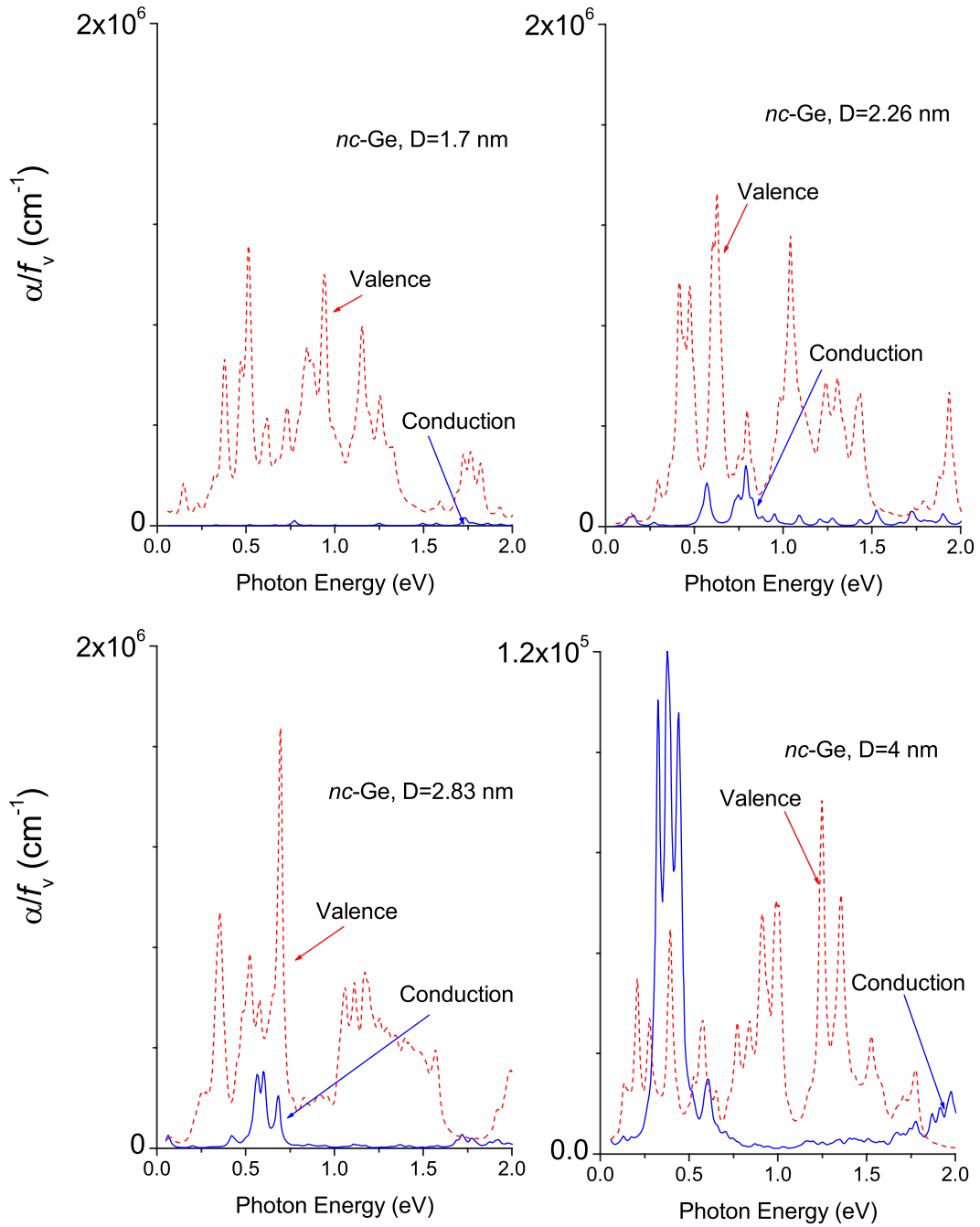

FIG. 13. (Color online) Same as Fig. 12 but for Ge NCs.
This provides us an opportunity to compare our results on the intraconduction band absorption in Si NCs. There is a good order-of-magnitude agreement. However, in contrast to our spectra in Fig. 12 which contains well-resolved peaks, they have registered a smooth spectrum, which has been attributed by the authors to the smearing out due to size and shape distribution within their NC ensemble. ${ }^{62}$

\section{E. Excited-state absorption}

Finally, we consider another intraband absorption process where the system is under a continuous interband optical pumping that creates electrons and holes with excess energy. We consider three different excitation wavelengths, 532, 355, and $266 \mathrm{~nm}$, which, respectively, correspond to the second, third, and fourth harmonics of the neodymium-doped yttrium aluminum garnet (Nd:YAG) laser at $1064 \mathrm{~nm}$. The initial states of the carriers after optical pumping are chosen to be at the pair of states with the maximum oscillator strength ${ }^{44}$ among interband transitions under the chosen excitation. The determined energies of these states are tabulated in Table II, where it can be observed that, in general, the excess energy is unevenly partitioned, mainly in favor of the conduction states. Once again, a Boltzmann averaging is used to get the

TABLE II. The excited-state energies of the carriers within the valence and conduction states under three different interband pump energies for $\mathrm{nc}-\mathrm{Si}$ and nc-Ge. The energies are given in $\mathrm{eV}$ and measured from the HOMO and LUMO, respectively.

\begin{tabular}{lccccc}
\hline \hline & \multicolumn{2}{c}{ nc-Si } & & \multicolumn{2}{c}{ nc-Ge } \\
\cline { 2 - 3 } \cline { 5 - 6 } Pump & $D=3 \mathrm{~nm}$ & $D=4 \mathrm{~nm}$ & & $D=3 \mathrm{~nm}$ & $D=4 \mathrm{~nm}$ \\
\hline 2.33 & $0.197,0.021$ & $0.021,0.551$ & & $0.103,0.643$ & $0.211,0.663$ \\
3.50 & $0.000,1.432$ & $0.316,1.440$ & & $0.400,1.508$ & $1.141,0.892$ \\
4.66 & $0.188,2.414$ & $0.713,2.218$ & & $0.511,2.551$ & $1.360,1.853$ \\
\hline \hline
\end{tabular}



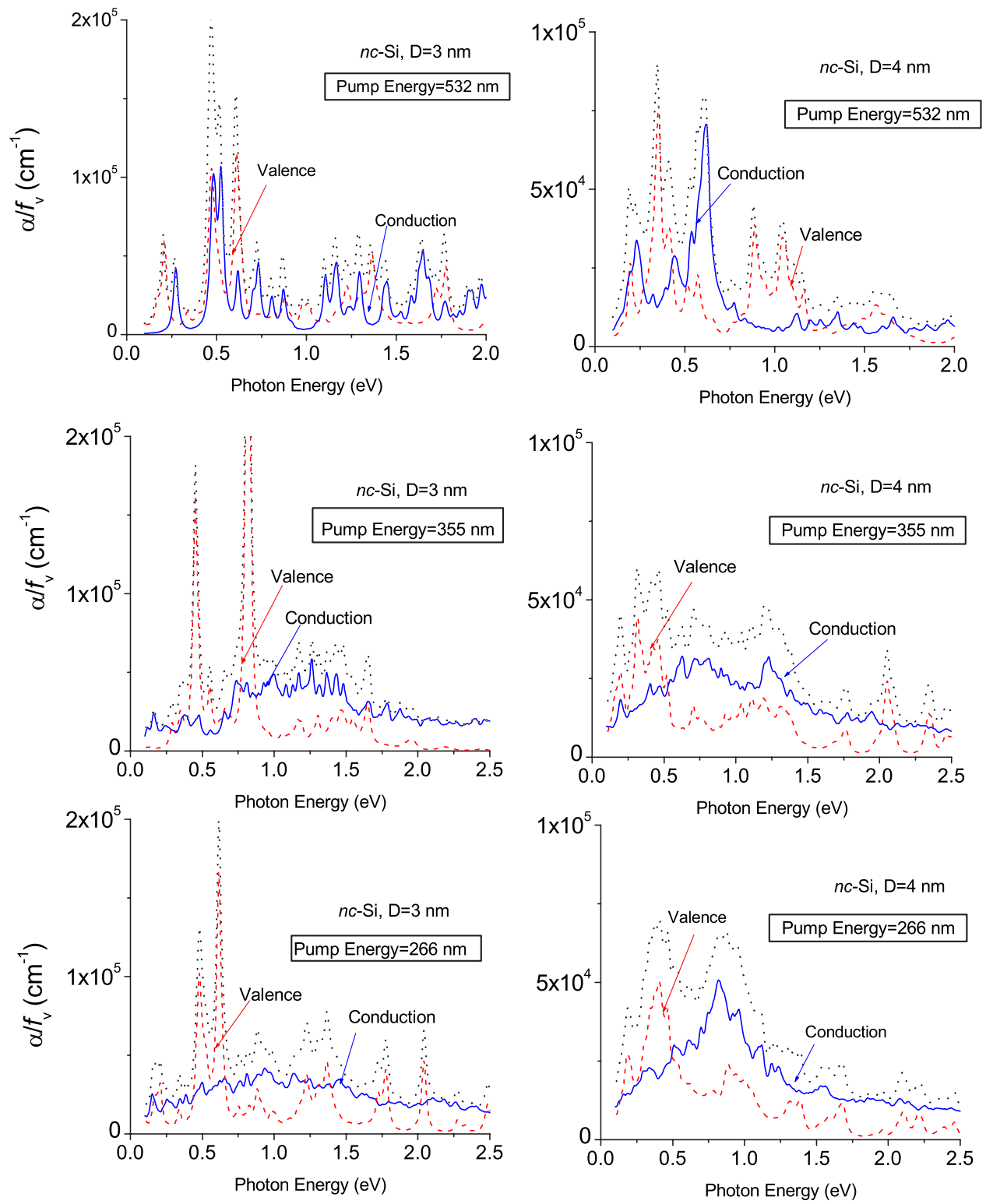

FIG. 14. (Color online) Excited-state absorption within valence and conduction states of Si NCs per excited carrier and at unity filling factor under three different optical pumping wavelengths of 532, 355, and $266 \mathrm{~nm}$. Dotted lines in black color refer to total absorption coefficients. Two different diameters are considered, 3 and $4 \mathrm{~nm}$. A Lorentzian broadening energy width of $30 \mathrm{meV}$ is used. contribution of states within the thermal energy neighborhood.

Considering 3 and $4 \mathrm{~nm}$ diameters, the results are shown in Figs. 14 and 15 for Si and Ge NCs, respectively. Note that the $532 \mathrm{~nm}$ excitation results are qualitatively similar to those in intraband absorption, cf. Figs. 12 and 13. This is expected on the grounds of small excess energy for this case. Some general trends can be extracted from these results. First of all, the conduction band absorption is, in general, smooth over a wide energy range. On the other hand, the valence band absorption contains pronounced absorption at several narrow energy windows mainly below $1 \mathrm{eV}$ and they get much weaker than the conduction band absorption in the remaining energies. As the excitation energy increases, the absorption coefficient per excited carrier, in general, decreases. In connection to silicon photonics, we should point out that the excited-state absorption is substantial including the important $1.55 \mu \mathrm{m}$ fiber optics communication wavelength. These results provide a more comprehensive picture than the reported experimental measurements, ${ }^{27-29}$ which are usually obtained at a single energy of the probe beam. Finally, it needs to be mentioned that for both intraband and excited-state absorptions displayed in Figs. 12-15, the high energy parts will be masked by the interband transition whenever it becomes energetically possible.

\section{CONCLUSIONS}

The subject of Si and Ge NCs has become an established research field. A fundamental process such as the direct photon absorption deserves further investigation from a number of perspectives. In this theoretical study, we consider the interband, intraband, and excited-state absorption in embedded $\mathrm{Si}$ and Ge NCs of various sizes. For this purpose, we developed an atomistic pseudopotential electronic structure tool, the results of which agree very well with the published data. It is further observed that the HOMO for both $\mathrm{Si}$ and Ge NCs and the LUMO for Si NCs display oscillations with respect to size among different representations of the $C_{3 v}$ point group to which these spherical NCs belong. Our de- 

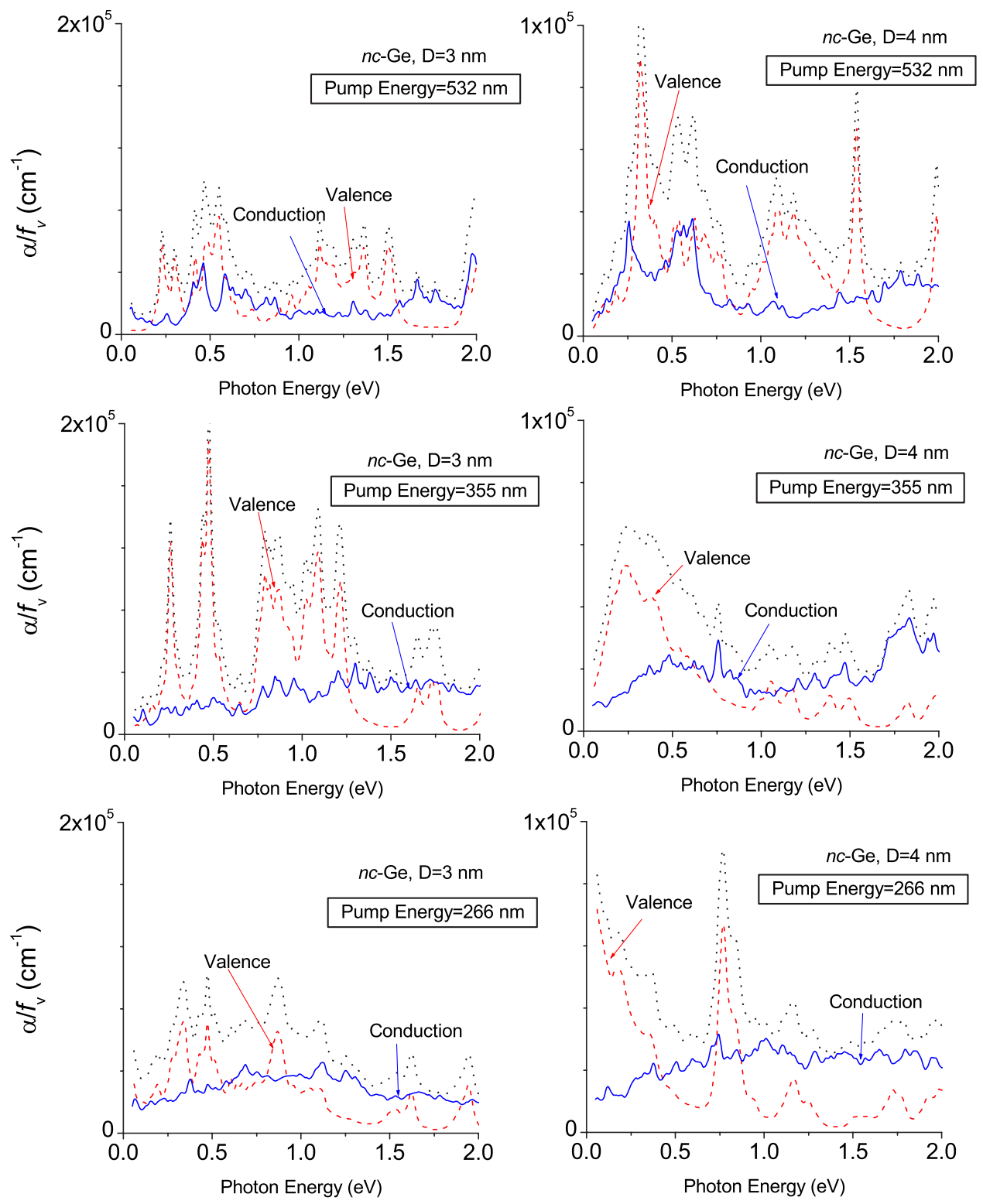

tailed investigation of the interband absorption reveals the importance of surface polarization effects that significantly reduce the absorption when included. This reduction is found to increase with decreasing $\mathrm{NC}$ size or with increasing permittivity mismatch between the NC core and the host matrix. These findings should be taken into account for applications where the absorption is desired to be either enhanced or reduced. For both NC types, the deviation from sphericity shows no pronounced effect on the interband absorption. Next, the intraband process is considered, which has potential applications on mid- and near-infrared photodetection. The intravalence band absorption is stronger compared to the intraconduction band especially below $1 \mathrm{eV}$. Finally, we demonstrate that optical pumping introduces a new degree of freedom to intraband absorption. This is studied under the title of excited-state absorption. Our major finding is that the excited-state absorption is substantial including the important $1.55 \mu \mathrm{m}$ fiber optics communication wavelength. Within the context of achieving gain and lasing in these NCs, excited-state absorption is a parasitic process; however, it can acquire a positive role in a different application.

\section{ACKNOWLEDGMENTS}

The author is grateful to Aykutlu Dâna for his suggestion of the intersubband absorption and its photodetector applications. This work has been supported by the European FP6 Project SEMINANO with Contract No. NMP4 CT2004 505285 and by the Turkish Scientific and Technical Council TÜBITAK with Project No. 106T048. The computational resources are supplied in part by TÜBİTAK through TR-Grid e-Infrastructure Project.

\section{APPENDIX: SOME TECHNICAL DETAILS ON THE LINEAR COMBINATION OF BULK BAND IMPLEMENTATION}

In this section, we first provide the details on the pseudopotential form factors of the bulk $\mathrm{Si}, \mathrm{Ge}$, and their associated wide band-gap matrices to substitute for $\mathrm{SiO}_{2}$. We use the 
TABLE III. Parameters of the pseudopotential form factors of $\mathrm{Si}, \mathrm{Ge}$, and their wide band-gap matrices. $a_{0}$ is the lattice constant (see text for the units).

\begin{tabular}{lccccccc}
\hline \hline & $\begin{array}{c}a_{0} \\
(\AA)\end{array}$ & $a_{1}$ & $a_{2}$ & $a_{3}$ & $a_{4}$ & $a_{5}$ & $a_{6}$ \\
\hline $\mathrm{Si}$ & 5.43 & 1.5708 & 2.2278 & 0.606 & -1.972 & 5.0 & 0.3 \\
Matrix Si & 5.43 & 1.5708 & 2.5 & 0.135 & -13.2 & 6.0 & 0.3 \\
Ge & 5.65 & 0.7158 & 2.3592 & 0.74 & -0.38 & 5.0 & 0.3 \\
Matrix Ge & 5.65 & 0.4101 & 2.7 & 0.07 & -2.2 & 5.0 & 0.3 \\
\hline \hline
\end{tabular}

local empirical pseudopotentials for Si and Ge developed by Friedel et $a{ }^{47}$ They use the following functional form for the pseudopotential form factor at a general wave number $q$ :

$$
V_{\mathrm{PP}}(q)=\frac{a_{1}\left(q^{2}-a_{2}\right)}{e^{a_{3}\left(q^{2}-a_{4}\right)}+1}\left[\frac{1}{2} \tanh \left(\frac{a_{5}-q^{2}}{a_{6}}\right)+\frac{1}{2}\right] .
$$

Using the parameters supplied in Table III, the pseudopotential form factors come out in Rydbergs and the wave number in the above equation should be taken in atomic units (1/ Bohr radius). Another important technical remark is about the EPM cutoff energies. We observed that even though the EPM band energies (i.e., eigenvalues) converge reasonably well with cut off energies as low as 5-10 Ry, the corresponding Bloch functions (i.e., eigenvectors) require sub- stantially higher values to converge. ${ }^{63}$ The results in this study are obtained using 14 and $16 \mathrm{Ry}$ for $\mathrm{Si}$ and $\mathrm{Ge}$, respectively.

Finally, some comments on the LCBB basis set construction are in order. We only employ the bulk bands of the core material. The bulk band indices are chosen from the four valence bands and the lowest three to four conduction bands; usually, these are not used in conjunction but separately for the NC valence and conduction states, respectively. The basis set is formed from a sampling over a three-dimensional rectangular grid in the reciprocal space centered around the $\Gamma$ point. Its extend is decided by the significant band extrema, such as conduction band minima of $\mathrm{Si}$ at the six equivalent $0.85 X$ points. The final LCBB basis set typically contains some 10000 members. *bulutay@fen.bilkent.edu.tr

${ }^{1}$ S. Ossicini, L. Pavesi, and F. Priolo, Light Emitting Silicon for Microphotonics (Springer-Verlag, Berlin, 2004).

${ }^{2}$ L. Pavesi, L. Dal Negro, C. Mazzoleni, G. Franzó, and F. Priolo, Nature (London) 408, 440 (2000).

${ }^{3}$ R. J. Walters, G. I. Bourianoff, and H. A. Atwater, Nat. Mater. 4, 143 (2005).

${ }^{4}$ H. Rong, A. Liu, R. Jones, O. Cohen, D. Hak, A. Fang, and M. Paniccia, Nature (London) 433, 292 (2005); H. Rong, R. Jones, A. Liu, O. Cohen, D. Hak, A. Fang, and M. Paniccia, ibid. 433, 725 (2005).

${ }^{5}$ M. V. Wolkin, J. Jorne, P. M. Fauchet, G. Allan, and C. Delerue, Phys. Rev. Lett. 82, 197 (1999).

${ }^{6}$ Z. Zhou, L. Brus, and R. Friesner, Nano Lett. 3, 163 (2003).

${ }^{7}$ M. Luppi and S. Ossicini, Phys. Rev. B 71, 035340 (2005).

${ }^{8}$ I. Sagnes, H. Halimaoui, G. Vincent, and P. A. Badoz, Appl. Phys. Lett. 62, 1155 (1993).

${ }^{9}$ M. Green, Third Generation Photovoltaics (Springer-Verlag, Berlin, 2006).

${ }^{10}$ S. Furukawa and T. Miyasato, Phys. Rev. B 38, 5726 (1988).

${ }^{11}$ Y. Kanemitsu, H. Uto, Y. Masimoto, and Y. Maeda, Appl. Phys. Lett. 61, 2187 (1992).

${ }^{12}$ S. Takeoka, M. Fujii, S. Hayashi, and K. Yamamoto, Phys. Rev. B 58, 7921 (1998).

${ }^{13}$ J. P. Wilcoxon, G. A. Samara, and P. N. Provencio, Phys. Rev. B 60, 2704 (1999).

${ }^{14}$ D. Kovalev, J. Diener, H. Heckler, G. Polisski, N. Künzner, and F. Koch, Phys. Rev. B 61, 4485 (2000).
${ }^{15}$ J. P. Wilcoxon, P. P. Provencio, and G. A. Samara, Phys. Rev. B 64, 035417 (2001).

${ }^{16}$ I. Vasiliev, S. Öğüt, and J. R. Chelikowsky, Phys. Rev. Lett. 86, 1813 (2001).

${ }^{17}$ H.-Ch. Weissker, J. Furthmüller, and F. Bechstedt, Phys. Rev. B 65, 155327 (2002).

${ }^{18}$ H.-Ch. Weissker, J. Furthmüller, and F. Bechstedt, Phys. Rev. B 65, 155328 (2002).

${ }^{19}$ H.-Ch. Weissker, J. Furthmüller, and F. Bechstedt, Phys. Rev. B 67, 245304 (2003).

${ }^{20}$ L. E. Ramos, H.-Ch. Weissker, J. Furthmüller, and F. Bechstedt, Phys. Status Solidi B 242, 3053 (2005).

${ }^{21}$ D. V. Melnikov and J. R. Chelikowsky, Solid State Commun. 127, 361 (2003); Phys. Rev. B 69, 113305 (2004).

${ }^{22}$ A. Tsolakidis and R. M. Martin, Phys. Rev. B 71, 125319 (2005).

${ }^{23}$ F. Trani, G. Cantele, D. Ninno, and G. Iadonisi, Phys. Rev. B 72, 075423 (2005).

${ }^{24}$ Y.-Y. Lin and J. Singh, J. Appl. Phys. 96, 1059 (2004).

${ }^{25}$ V. Ryzhii, I. Khmyrova, V. Mitin, M. Stroscio, and M. Willander, Appl. Phys. Lett. 78, 3523 (2001).

${ }^{26}$ A. V. Malko, A. A. Mikhailovsky, M. A. Petruska, J. A. Hollingsworth, and V. I. Klimov, J. Phys. Chem. B 108, 5250 (2004).

${ }^{27}$ R. G. Elliman, M. J. Lederer, N. Smith, and B. Luther-Davies, Nucl. Instrum. Methods Phys. Res. B 206, 427 (2003).

${ }^{28}$ F. Trojánek, K. Neudert, M. Bittner, and P. Malý, Phys. Rev. B 72, 075365 (2005).

${ }^{29}$ M. Forcales, N. J. Smith, and R. G. Elliman, J. Appl. Phys. 100, 014902 (2006). 
${ }^{30}$ A. Zunger, Phys. Status Solidi A 190, 467 (2002).

${ }^{31}$ R. M. Martin, Electronic Structure (Cambridge University Press, Cambridge, 2004).

${ }^{32}$ S. Öğüt, J. R. Chelikowsky, and S. G. Louie, Phys. Rev. Lett. 79, 1770 (1997).

${ }^{33}$ C. Delerue and M. Lannoo, Nanostructures: Theory and Modelling (Springer-Verlag, Berlin, 2004).

${ }^{34}$ S. Y. Ren, Phys. Rev. B 55, 4665 (1997); S. Y. Ren, Solid State Commun. 102, 479 (1997).

${ }^{35}$ Y. M. Niquet, G. Allan, C. Delerue, and M. Lannoo, Appl. Phys. Lett. 77, 1182 (2000).

${ }^{36}$ L.-W. Wang and A. Zunger, J. Chem. Phys. 100, 2394 (1994).

${ }^{37}$ L.-W. Wang, A. Franceschetti, and A. Zunger, Phys. Rev. Lett. 78, 2819 (1997).

${ }^{38}$ L.-W. Wang and A. Zunger, Phys. Rev. B 59, 15806 (1999).

${ }^{39}$ D. Ninno, K. B. Wong, M. A. Gell, and M. Jaros, Phys. Rev. B 32, 2700 (1985).

${ }^{40}$ D. Ninno, M. A. Gell, and M. Jaros, J. Phys. C 19, 3845 (1986).

${ }^{41}$ S. Botti and L. C. Andreani, Phys. Rev. B 63, 235313 (2001).

${ }^{42}$ S. Botti, N. Vast, L. Reining, V. Olevano, and L. C. Andreani, Phys. Rev. B 70, 045301 (2004).

${ }^{43}$ F. Chirico, A. DiCarlo, and P. Lugli, Phys. Rev. B 64, 045314 (2001).

${ }^{44}$ C. Bulutay, Physica E (Amsterdam) 38, 112 (2007).

${ }^{45}$ F. Iacona, G. Franzó, and C. Spinella, J. Appl. Phys. 87, 1295 (2000).

${ }^{46} \mathrm{An}$ intermediate value between 0 and 1 can be used for the alloys or modeling the interface region. However, in this work, we set them to either 1 or 0 .
${ }^{47}$ P. Friedel, M. S. Hybertsen, and M. Schlüter, Phys. Rev. B 39, 7974 (1989).

${ }^{48}$ J. R. Chelikowsky, Solid State Commun. 22, 351 (1977).

${ }^{49}$ J. D. Jackson, Classical Electrodynamics, 2nd ed. (Wiley, New York, 1975).

${ }^{50}$ F. Trani, D. Ninno, and G. Iadonisi, Phys. Rev. B 75, 033312 (2007).

${ }^{51}$ G. D. Mahan, Phys. Rev. B 74, 033407 (2006).

${ }^{52}$ B. Delley and E. F. Steigmeier, Phys. Rev. B 47, 1397 (1993).

${ }^{53}$ F. A. Reboredo and A. Zunger, Phys. Rev. B 63, 235314 (2001).

${ }^{54}$ G. Onida, L. Reining, and A. Rubio, Rev. Mod. Phys. 74, 601 (2002).

${ }^{55}$ H. Ehrenreich and M. H. Cohen, Phys. Rev. 115, 786 (1959).

${ }^{56}$ M. S. Hybertsen, Phys. Rev. Lett. 72, 1514 (1994).

${ }^{57}$ F. A. Reboredo, A. Franceschetti, and A. Zunger, Phys. Rev. B 61, 13073 (2000).

${ }^{58}$ L.-W. Wang and A. Zunger, J. Phys. Chem. 98, 2158 (1994).

${ }^{59}$ C. S. Garoufalis, A. D. Zdetsis, and S. Grimme, Phys. Rev. Lett. 87, 276402 (2001).

${ }^{60}$ C. Delerue, M. Lannoo, and G. Allan, Phys. Rev. Lett. 84, 2457 (2000).

${ }^{61}$ J. S. de Sousa, J.-P. Leburton, V. N. Freire, and E. F. da Silva, Jr., Appl. Phys. Lett. 87, 031913 (2005).

${ }^{62}$ A. Mimura, M. Fujii, S. Hayashi, D. Kovalev, and F. Koch, Phys. Rev. B 62, 12625 (2000).

${ }^{63}$ C. Bulutay, Turk. J. Phys. 30, 287 (2006). 